\title{
Modélisation thermomécanique de structures réfractaires comportant des joints de dilatation
}

\author{
Alain Gasser $^{a}$, Karine Terny-Rebeyrotte et Philippe Boisse
}

Laboratoire de Mécanique des Systèmes et des Procédés (UMR 8106 CNRS-ENSAM-Université d'Orléans), Polytech’Orléans, 8 rue Léonard de Vinci, 45072 Orléans Cedex 2, France

Reçu le 3 octobre 2003, accepté le 15 avril 2004

\begin{abstract}
Résumé - Les joints de dilatation jouent un rôle important dans les garnissages réfractaires. Ils permettent de diminuer les contraintes durant la montée en température. Il est alors nécessaire de les prendre en compte lors d'un calcul mécanique. Dans le cas où le garnissage est une maçonnerie (composée de briques), il n'est pas possible de modéliser chaque brique et joint. La solution proposée est de remplacer la maçonnerie par un matériau qui aurait le même comportement. Du fait qu'il est difficile de réaliser des essais sur un ensemble de briques (dans le but d'identifier les paramètres du matériau équivalent), ces chargements ont été simulés sur une cellule élémentaire en utilisant un modèle développé à l'échelle des composants, à laquelle les joints sont représentés par un contact. Une identification inverse a permis d'obtenir les paramètres d'un matériau équivalent simplifié qui a été validé à l'aide d'un essai thermomécanique sur une structure réelle.
\end{abstract}

Mots clés : Joints / garnissages réfractaires / approche multi-échelles / identification inverse / éléments finis / calculs thermomécaniques

\begin{abstract}
Thermomechanical modelling of refractory structures including expansion joints. Dilatation joints play an important role in refractory linings. They allow to decrease stresses during heating. It is then necessary to take them into account in a mechanical analysis. In the case where the lining is a masonry (made up of bricks), it is not possible to model each brick and joint. The proposed solution is to replace the masonry by a material that has the same behaviour. Since it is difficult to perform experimental tests on a set of bricks (in the aim to identify the parameters of the equivalent material), these loads were simulated on an elementary cell using a model developed at the local scale of the components, where the joints are represented as a contact. Inverse identification has allowed to obtain the parameters of a simplified equivalent material that was validated by a thermomechanical test on a real structure.
\end{abstract}

Key words: Joints / refractory linings / multi-scale approach / inverse identification / finite elements / thermomechanical analyses

\section{Introduction}

Les garnissages réfractaires permettent de protéger les structures métalliques des produits chauds qu'elles contiennent, comme le charbon durant sa combustion $\left(850{ }^{\circ} \mathrm{C}\right)$ dans des centrales thermiques [1], ou l'acier liquide $\left(1650{ }^{\circ} \mathrm{C}\right.$ dans des poches à acier) dans l'industrie sidérurgique $[2,3]$. À cause de la différence des coefficients de dilatation thermique entre l'acier et les matériaux réfractaires, des contraintes importantes apparaissent dans les réfractaires (pendant la montée en température) qui peuvent conduire à la fissuration du

\footnotetext{
${ }^{a}$ Auteur correspondant : alain.gasser@univ-orleans.fr
}

garnissage. Des calculs éléments finis de la structure globale peuvent apporter une aide lors de la conception afin de diminuer ces contraintes [4-7]. Dans certains cas, comme pour la couche d'usure (qui est en contact direct avec l'acier liquide) d'une poche à acier, le garnissage réfractaire est constitué d'une maçonnerie de briques (Fig. 1). Si cette maçonnerie est modélisée par une entité continue ayant le comportement du matériau de la brique, les contraintes calculées seront surestimées. En effet, au début de la montée en température, les joints entre briques (Fig. 1) vont se fermer (à cause de la dilatation thermique), dans un premier temps, sans augmentation des contraintes. Ainsi, il est très important de prendre en compte ces joints lors d'un calcul éléments finis. 


\section{Nomenclature}

\begin{tabular}{|c|c|}
\hline$E_{i}$ & module d'Young dans la direction $i$ \\
\hline$G_{12}$ & module de cisaillement dans le plan 12 \\
\hline$\nu_{12}$ & coefficient de Poisson dans le plan 12 \\
\hline$\sigma_{1}^{0}$ & $\begin{array}{l}\text { seuil de contrainte de fermeture des joints dans la direction } 1 \\
\text { quand les joints sont ouverts dans la direction } 2\end{array}$ \\
\hline$\sigma_{1}^{\mathrm{f}}$ & $\begin{array}{l}\text { seuil de contrainte de fermeture des joints dans la direction } 1 \\
\text { quand les joints sont fermés dans la direction } 2\end{array}$ \\
\hline$\sigma_{2}$ & seuil de contrainte de fermeture des joints dans la direction 2 \\
\hline
\end{tabular}
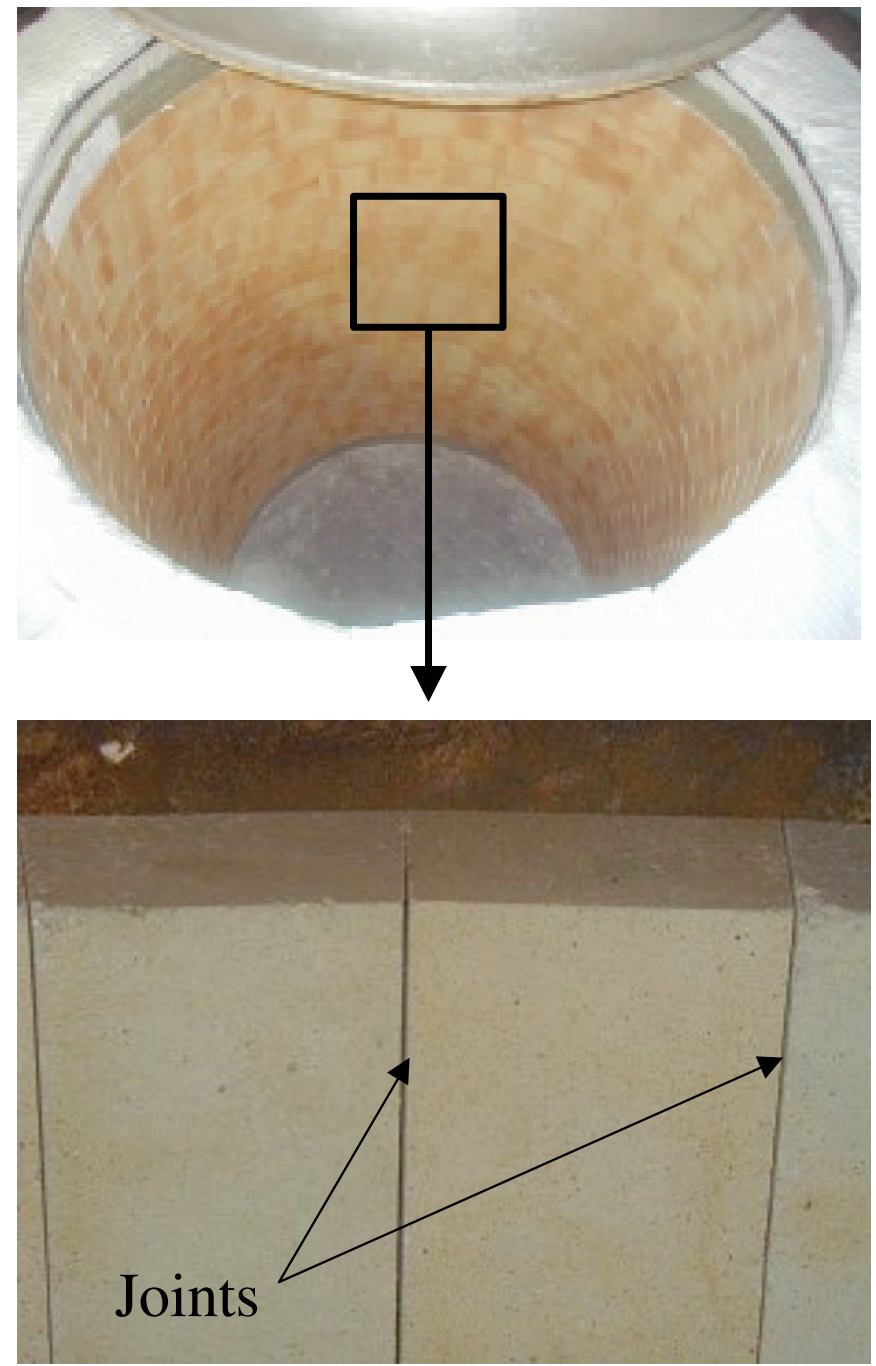

Fig. 1. Maçonnerie réfractaire dans une poche à acier (photos Sollac).

Un grand nombre d'études ont été réalisées pour modéliser le comportement de maçonneries de génie civil ([8-12] par exemple). Elles utilisent différentes techniques d'homogénéisation, mais presque toutes concernent des maçonneries comportant un mortier entre les briques, et ceci souvent à température ambiante. Peu d'études [13,14] (par exemple) concernent des maçonneries avec joints secs (sans mortier). Notre but est de développer un modèle

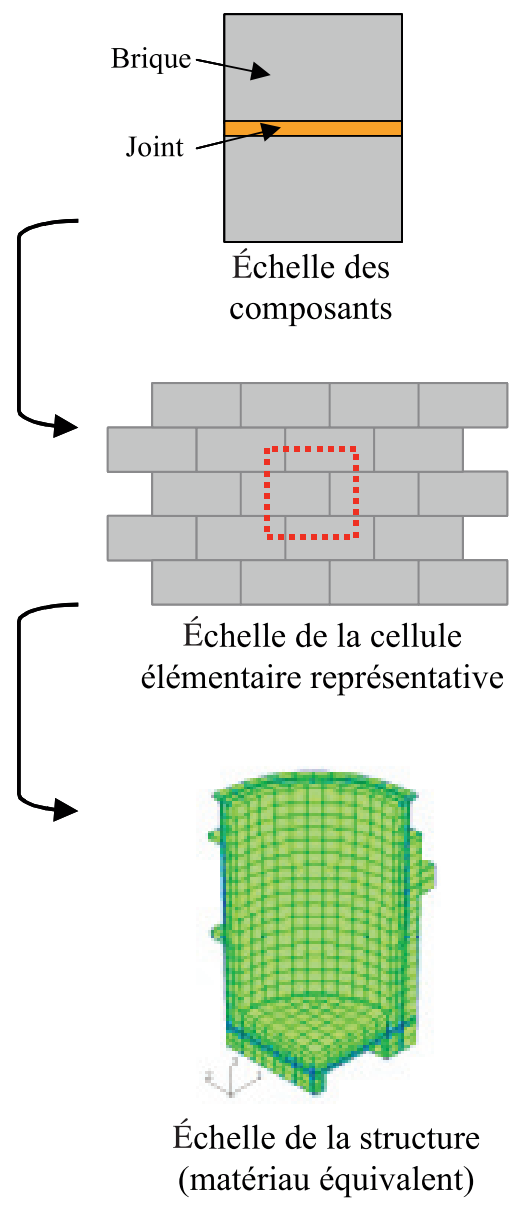

Fig. 2. Les différentes échelles du problème.

pour ce type de maçonnerie, qui pourra être employé à haute température.

Du fait qu'il n'est pas possible de modéliser chaque brique et chaque joint de la maçonnerie (le nombre de briques étant trop important), l'approche développée dans cette étude est de remplacer la maçonnerie par un matériau qui aurait un comportement équivalent à un ensemble de briques. Afin d'identifier les paramètres de ce matériau équivalent, une approche à plusieurs échelles (Fig. 2) a été utilisée. En effet, pour obtenir les caractéristiques mécaniques et thermiques du matériau équivalent, il est nécessaire de réaliser un certain nombre d'essais, à différentes températures, sur une maçonnerie : compression, traction, cisaillement, par exemple. Mais il 
est facile de voir que ces essais sont difficiles à réaliser. Ainsi, l'idée est de simuler ces différents chargements sur une cellule élémentaire représentative à l'aide d'un modèle défini à l'échelle inférieure (appelée échelle locale) qui est l'échelle des composants élémentaires (briques et joints). L'avantage de cette approche est que seuls les comportements des briques et des joints sont nécessaires, et qu'il est plus facile de réaliser des essais sur des briques et des joints que sur un ensemble de plusieurs briques.

Le modèle présenté dans cette étude est une première approche, en 2D, avec un matériau équivalent simplifié.

\section{Modélisation du comportement des constituants élémentaires}

Les constituants élémentaires d'une maçonnerie sont les briques et les joints. Ce paragraphe décrit leur modélisation. La maçonnerie étudiée est constituée de briques en bauxite cuites (surface $200 \times 100 \mathrm{~mm}^{2}$ ).

\subsection{Comportement des briques}

Dans une première approche et dans un but de simplification, le comportement de la brique est supposé élastique linéaire isotrope. Les paramètres mécaniques sont obtenus par un essai de compression. Les paramètres sont :

- le module d'Young : $7000 \mathrm{MPa}$,

- le coefficient de Poisson : 0,2,

- le coefficient de dilatation thermique : $7 \times 10^{-6} \mathrm{~K}^{-1}$.

\subsection{Comportement des joints}

Les joints sont modélisés par un contact entre deux solides séparés par une distance correspondant à l'épaisseur du joint. Pour ce modèle, les données suivantes sont nécessaires :

- l'épaisseur des joints,

- le comportement tangentiel du contact,

- le comportement normal du contact.

\subsubsection{Comportement tangentiel}

Le comportement tangentiel est donné par un modèle de frottement de Coulomb. Le coefficient de frottement est mesuré par un dispositif très simple (plan à inclinaison variable) qui permet de déterminer l'angle de début de glissement entre deux briques. Le coefficient de frottement obtenu pour des briques en bauxite est de 0,75 .

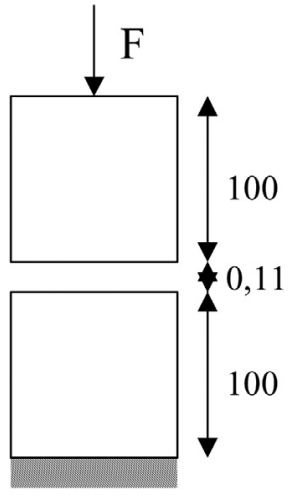

Fig. 3. Compression de deux briques en contact (dimensions en $\mathrm{mm}$ ).

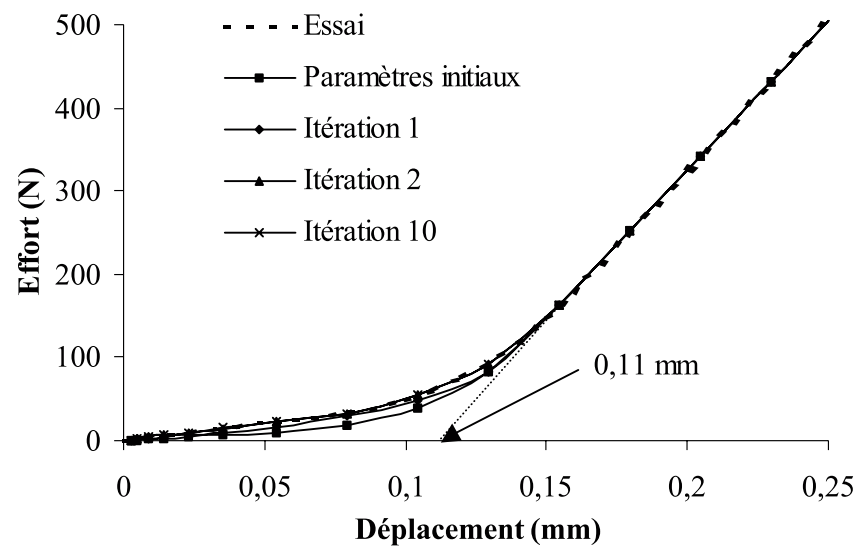

Fig. 4. Comparaison essai/simulation d'une compression de deux briques (avec les différentes itérations de la méthode inverse).

\subsubsection{Comportement normal}

Le comportement normal donne l'évolution de la pression de contact en fonction de la distance entre deux briques. Cette pression est nulle lorsque la distance est supérieure ou égale à l'épaisseur du joint, et est maximale lorsque la distance est nulle. Pour obtenir la loi complète (entre ces deux valeurs extrêmes), un essai de compression a été réalisé sur deux briques (Fig. 3). La courbe effort/déplacement (Fig. 4) est composée de deux parties :

- la première (non-linéaire) correspond à un écrasement du joint,

- la deuxième (linéaire) correspond à la rigidité de la brique.

Il est possible d'obtenir une estimation de l'épaisseur du joint à l'aide de cette courbe en considérant l'intersection du prolongement de la partie linéaire (correspondant à la rigidité de la brique) avec l'axe des abscisses (voir Fig. 4). On obtient $0,11 \mathrm{~mm}$.

La loi de comportement normal du contact est identifiée à l'aide d'une méthode inverse. Le principe est le suivant. L'essai de compression sur deux briques en contact est simulé par éléments finis, en utilisant des paramètres initiaux quelconques pour la loi de contact normal. 


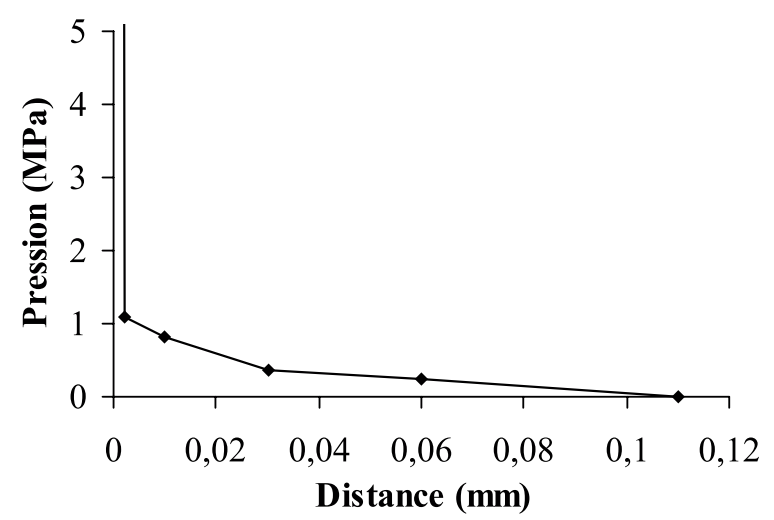

Fig. 5. Comportement normal du contact.

Une erreur est définie entre les courbes expérimentales et simulées. Cette erreur est minimisée, et les paramètres optimisés, en utilisant l'algorithme d'optimisation de Levenberg-Marquardt [15-18]. Cette méthode est itérative (Fig. 4). La figure 5 montre le résultat de l'identification inverse.

\subsubsection{Mesures optiques}

Une autre méthode pour obtenir la loi de comportement normal du contact a été réalisée par mesures optiques (Fig. 6) afin de déterminer directement la fermeture progressive des joints (i.e. l'épaisseur du joint en fonction de l'effort). Plusieurs images du joint sont prises à l'aide d'une caméra à différents moments du chargement. Une méthode de corrélation d'images [19-21] permet de déterminer le champ de déplacement (Fig. 7a). La différence de déplacement entre les deux briques au niveau du joint donne l'écrasement du joint en fonction de la force appliquée. La figure $7 \mathrm{~b}$ montre le résultat pour des mesures réalisées à trois emplacements différents du joint. La relative dispersion des courbes montre que l'écrasement du joint n'est pas seulement dû à l'écrasement des rugosités, mais aussi aux défauts de forme des briques (non planéité ou non parallélisme des faces). On peut même rajouter une autre origine à l'ouverture des joints : lors de la construction, certaines briques ne sont pas parfaitement placées dans la maçonnerie. Tous ces défauts doivent être pris en compte pour déterminer l'épaisseur initiale des joints dans une maçonnerie réelle.

\section{Comportement du matériau équivalent}

Le but de ce paragraphe est de déterminer les caractéristiques du matériau qui doit avoir un comportement équivalent à celui de la maçonnerie. Pour cela, il est nécessaire d'obtenir le comportement d'un ensemble de briques, en utilisant le modèle à l'échelle locale (des briques et des joints) décrit ci-dessus.

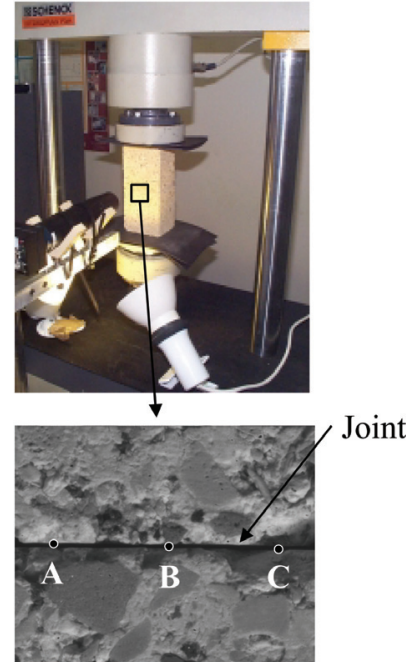

Fig. 6. Montage de mesure optique afin de déterminer l'écrasement du joint au cours d'un essai de compression sur deux briques.

\subsection{Simulations de différents chargements sur la cellule élémentaire}

En utilisant les comportements des joints et des briques décrits à la section 2, il est possible de simuler le comportement d'une cellule élémentaire représentative (CER). Cette cellule est présentée figure 8. Elle représente la périodicité de la structure, et n'a pas de joints sur ses bords (sinon le contact ne pourrait pas être pris en compte). Plusieurs chargements sont simulés :

- des compressions dans les directions 1 et 2 ,

- des cisaillements dans les «directions » 1 et 2 sous compression transverse,

- une traction dans la direction 1 sous compression transverse (une traction dans la direction 2 n'est pas simulée car la rigidité est nulle).

Les simulations de chargements de compression dans les directions 1 et 2 (Fig. 9) montrent que le comportement est non-linéaire dans la première partie où les joints se ferment progressivement, puis devient linéaire lorsque ces joints sont fermés. Les deux courbes (dans la direction de compression) sont différentes car il y a deux fois plus de joints dans la direction 2 que dans la direction 1 .

Un chargement de traction peut être réalisé uniquement dans la direction 1 (dans la direction 2, la rigidité serait nulle), à la condition qu'un chargement de compression soit appliqué au préalable dans la direction 2 (Fig. 10). De plus, la valeur de ce chargement transverse a une influence importante sur la surface du cycle de traction (chargement et déchargement, Fig. 10b). Il peut être observé :

- une première partie du cycle approximativement élastique, mais avec une rigidité inférieure à celle de la brique seule,

- une seconde partie horizontale correspondant au glissement entre briques. Le début du glissement dépend de la valeur du déplacement transverse imposé, 


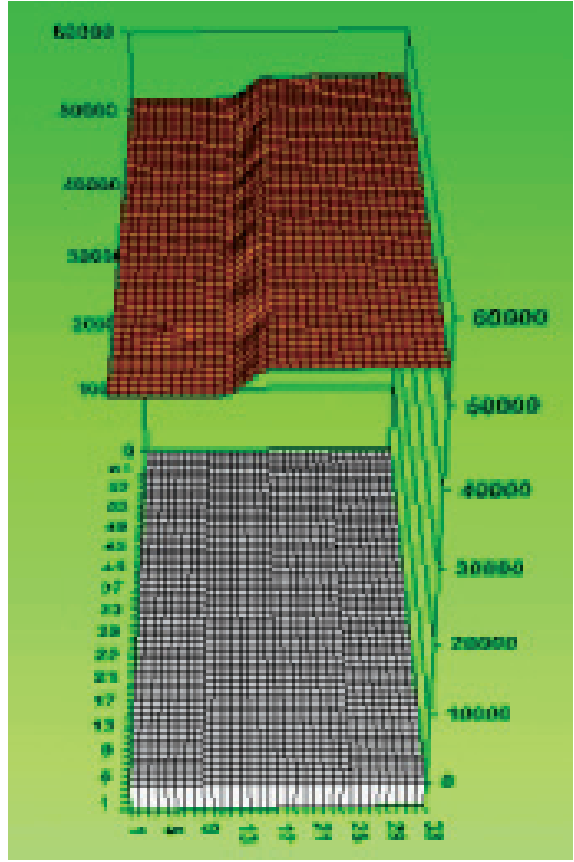

(a)

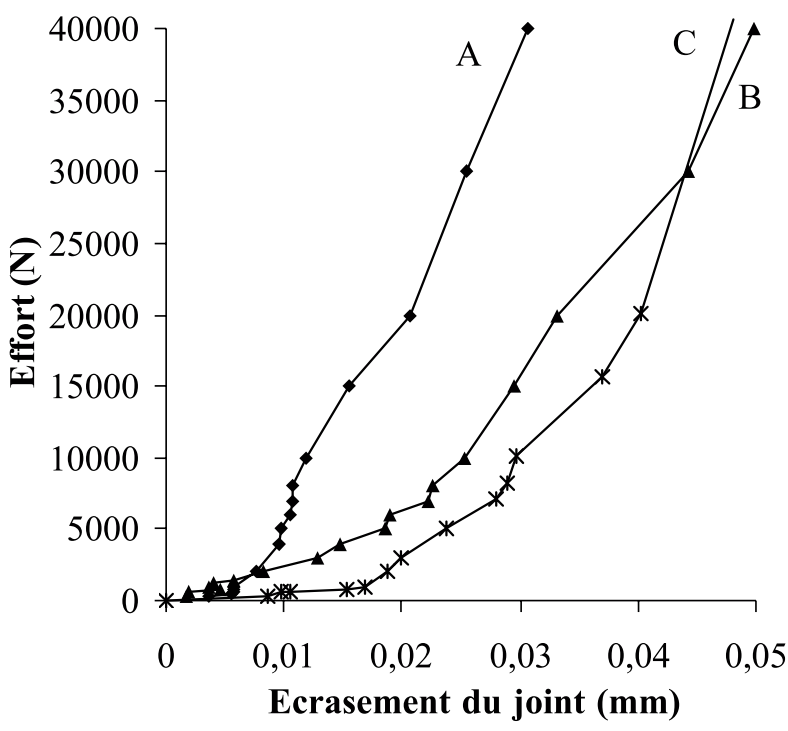

(b)

Fig. 7. Détermination de l'écrasement d'un joint par méthode optique : (a) champ de déplacement (en pixel) autour du joint, (b) écrasement du joint (en trois points différents, A, B et C, voir Fig. 6) en fonction de l'effort appliqué.

- une troisième partie correspondant au début du déchargement, avec arrêt du glissement, et ayant la même rigidité que la première partie,

- une quatrième partie, également horizontale, qui correspond à nouveau à du glissement. On peut noter que cette quatrième partie n'est pas symétrique par rapport à la seconde car le glissement ne commence pas

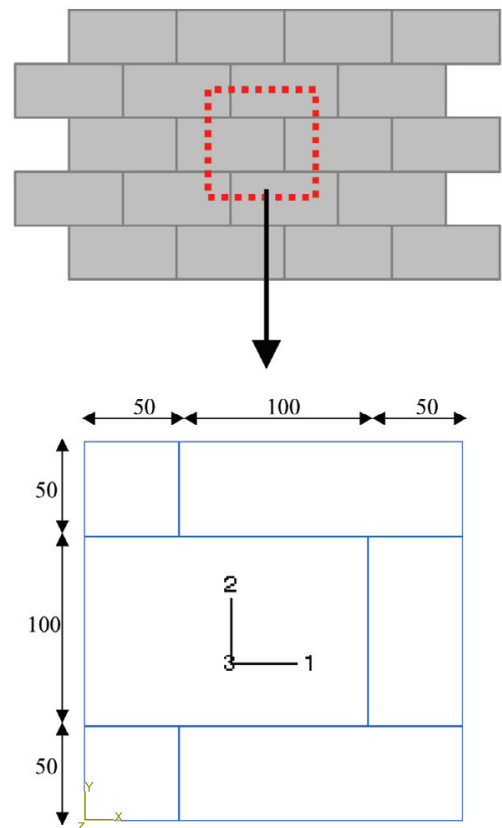

Fig. 8. Cellule élémentaire représentative (dimensions en $\mathrm{mm})$.

pour la même valeur du déplacement, du fait que les efforts transverses (RF2, Fig. 10b) sont différents au cours du chargement et du déchargement.

Les simulations de chargements de cisaillement doivent également être réalisés sous compression transverse. Le chargement de cisaillement est obtenu en bloquant une face et en imposant les déplacements sur la face opposée, dans la direction de cette même face. Il y a deux possibilités : soit dans la direction 1 (Fig. 11a), soit dans la direction 2 (Fig. 12a). Les résultats sont différents (Figs. $11 \mathrm{~b}$ et $12 \mathrm{~b}$ ). De plus, les rigidités initiales pour un même chargement avec des valeurs de compression transverse différentes ne sont pas identiques. Ces deux remarques augmentent la difficulté d'identification d'un matériau équivalent. Durant ces chargements de cisaillement, des hystérésis dues au glissement peuvent être observées, mais plus faibles qu'en traction.

\subsection{Matériau équivalent simplifié}

Un premier matériau équivalent simplifié est proposé. Son comportement a été choisi élastique orthotrope nonlinéaire. Deux simplifications ont été ajoutées :

- un possible glissement entre briques (qui est à l'origine du comportement anélastique) n'est pas pris en compte,

- une fermeture progressive des joints n'est pas considérée. Les joints sont soit ouverts, soit fermés, sans cas intermédiaire.

Dans ce cas, les neuf coefficients d'orthotropie dépendent de l'état (ouvert ou fermé) des joints (par la suite, joints ouverts dans la direction 1 signifiera que 


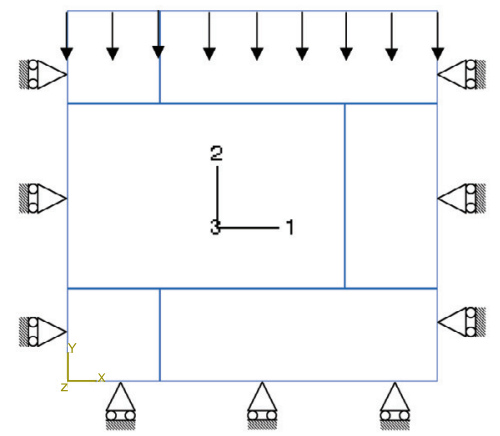

(a)

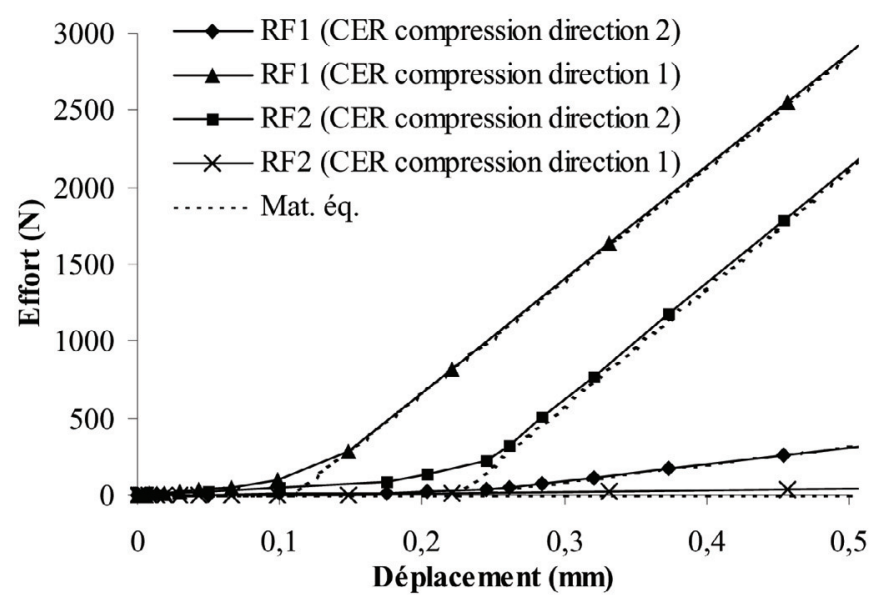

(b)

Fig. 9. (a) Conditions aux limites pour la simulation d'un chargement de compression dans la direction 2 sur la cellule élémentaire ; (b) comparaison des courbes obtenues avec la cellule élémentaire (CER) et avec le matériau équivalent (Mat. Éq.) pour des chargements de compression dans les deux directions principales (forces de réaction $\mathrm{RF} 1$ et $\mathrm{RF} 2$ dans les directions 1 et 2 , respectivement, en fonction des déplacements imposés $u_{1}$ ou $u_{2}$ ).

les joints perpendiculaires à la direction 1 sont ouverts). Quatre cas doivent être considérés :

- les joints sont ouverts dans les deux directions,

- les joints sont fermés dans les deux directions,

- les joints sont ouverts dans la direction 1 et fermés dans la direction 2 ,

- les joints sont ouverts dans la direction 2 et fermés dans la direction 1.

Les deux premiers cas sont les plus simples. Quand les joints sont ouverts dans les deux directions, la rigidité est nulle. Quand les joints sont fermés dans les deux directions, la rigidité est celle des briques. Pour les deux cas restants (joints ouverts dans une direction, fermés dans l'autre), l'identification inverse des caractéristiques mécaniques est réalisée à l'aide des chargements de compression, traction et cisaillement (sous compression transverse) réalisés sur la cellule élémentaire

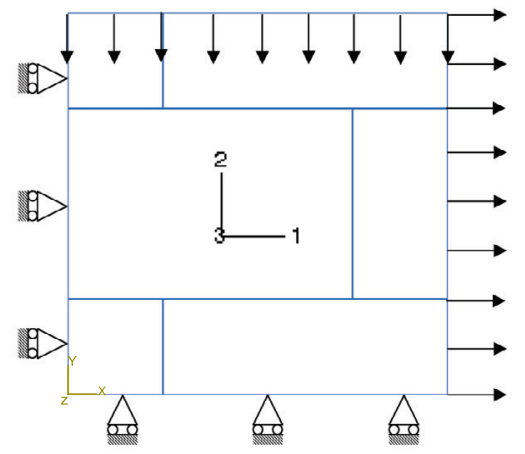

(a)

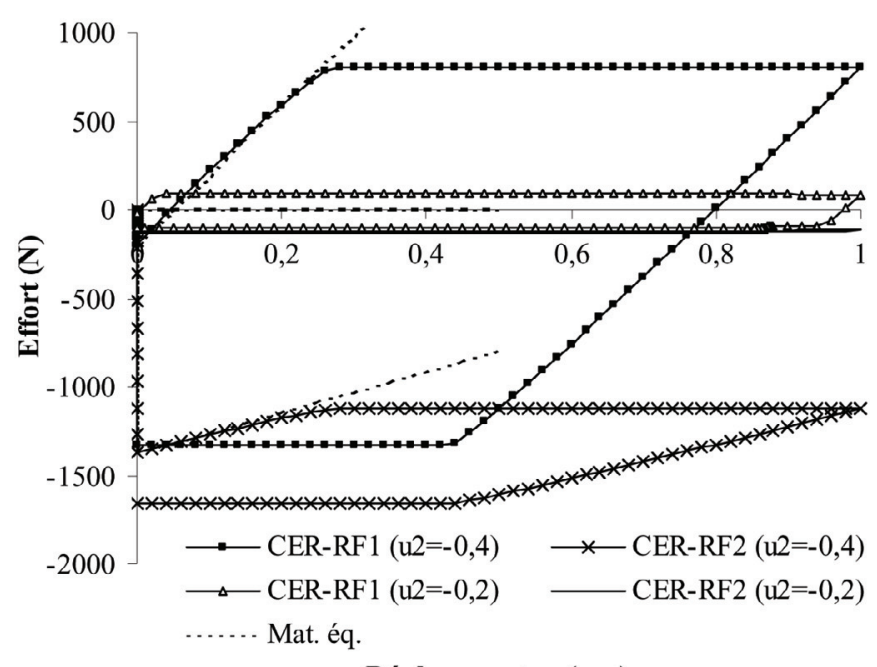

Déplacement $u_{1}(\mathrm{~mm})$

(b)

Fig. 10. Chargement de traction dans la direction 1 (sous compression transverse) : (a) conditions aux limites pour la simulation sur la cellule élémentaire; (b) comparaison des courbes obtenues avec la cellule élémentaire (CER) et avec le matériau équivalent (Mat. Éq.) pour deux valeurs de compression transverse différentes $u_{2}$ de 0,2 et $0,4 \mathrm{~mm}$ (RF1 et RF2 sont les forces de réaction dans les directions 1 et 2 , respectivement).

(présentés Sect. 3.1). En ce qui concerne le module de cisaillement $G_{12}$, c'est la valeur moyenne qui a été choisie, indépendamment de la « direction de cisaillement $»$ ou de la valeur de compression transverse.

Les coefficients thermiques et thermomécaniques (conductivité, chaleur spécifique, dilatation thermique) sont pris, dans une première approximation, comme étant égaux à ceux des briques.

Les résultats finaux sont présentés dans le tableau 1. $\mathrm{Du}$ fait que des valeurs nulles ne sont pas admises dans un calcul éléments finis pour les modules d'Young et de cisaillement, elles sont remplacées par des valeurs très faibles (1 MPa). 


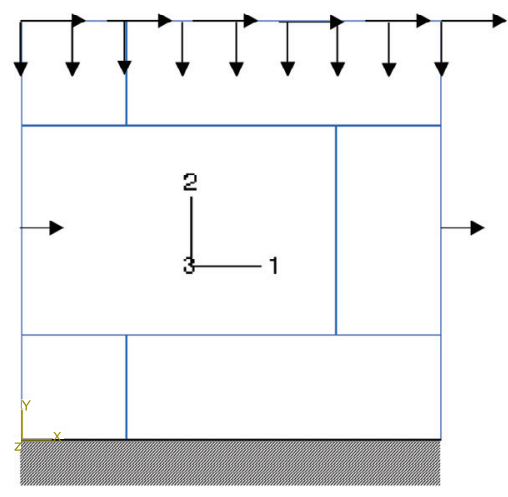

(a)

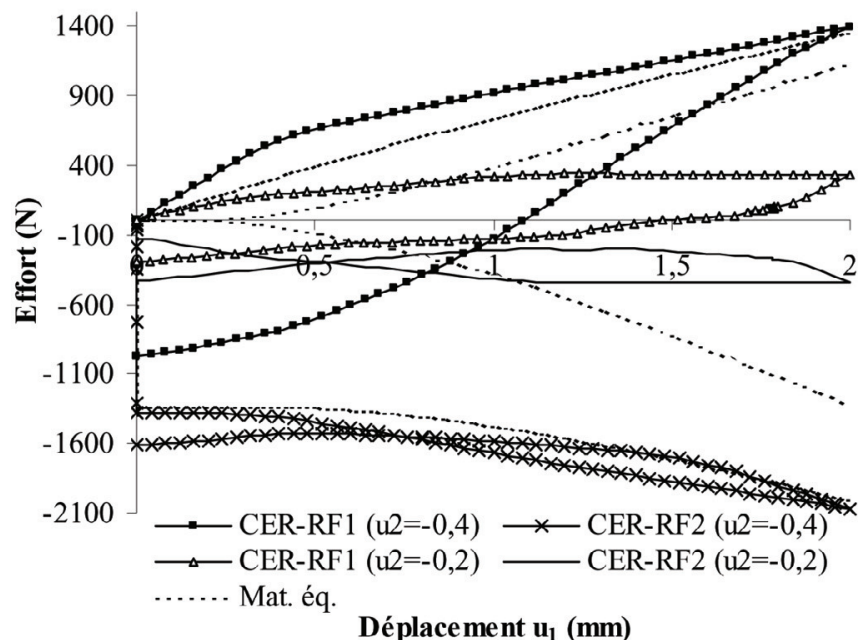

(b)

Fig. 11. Chargement de cisaillement dans la direction 1 (sous compression transverse) : (a) conditions aux limites pour la simulation sur la cellule élémentaire; (b) comparaison des courbes obtenues avec la cellule élémentaire (CER) et avec le matériau équivalent (Mat. Éq.) pour deux valeurs de compression transverse différentes $u_{2}$ de 0,2 et $0,4 \mathrm{~mm}$ (RF1 et $\mathrm{RF} 2$ sont les forces de réaction dans les directions 1 et 2 , respectivement).

Afin de déterminer si les joints sont ouverts ou fermés, un critère en contrainte est utilisé. Dans la direction 2 , du fait que les joints traversent toute la longueur de la cellule élémentaire, la contrainte nécessaire pour fermer ces joints (appelée $\sigma_{2}$ ) est indépendante de l'état des joints dans la direction 1. Par contre, la contrainte nécessaire à fermer les joints dans la direction 1 dépend de l'état des joints dans la direction 2. Cette contrainte est notée $\sigma_{1}^{0}$ lorsque les joints sont ouverts dans la direction 2 , et $\sigma_{1}^{\mathrm{f}}$ quand ils sont fermés.

Les valeurs de ces trois contraintes seuils sont déterminées à l'aide de la méthode inverse à partir de deux chargements de compression, en utilisant le changement de pente des courbes de comportement transverse

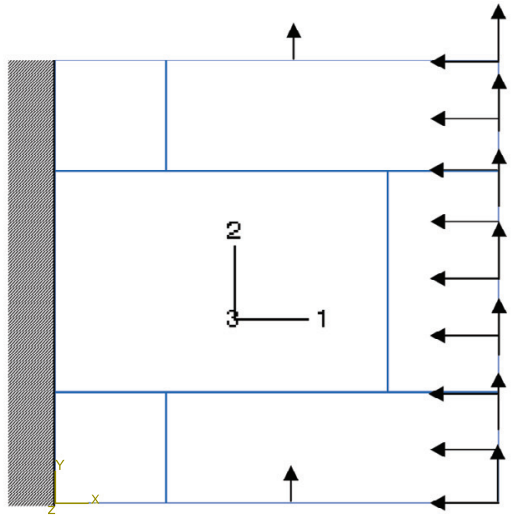

(a)

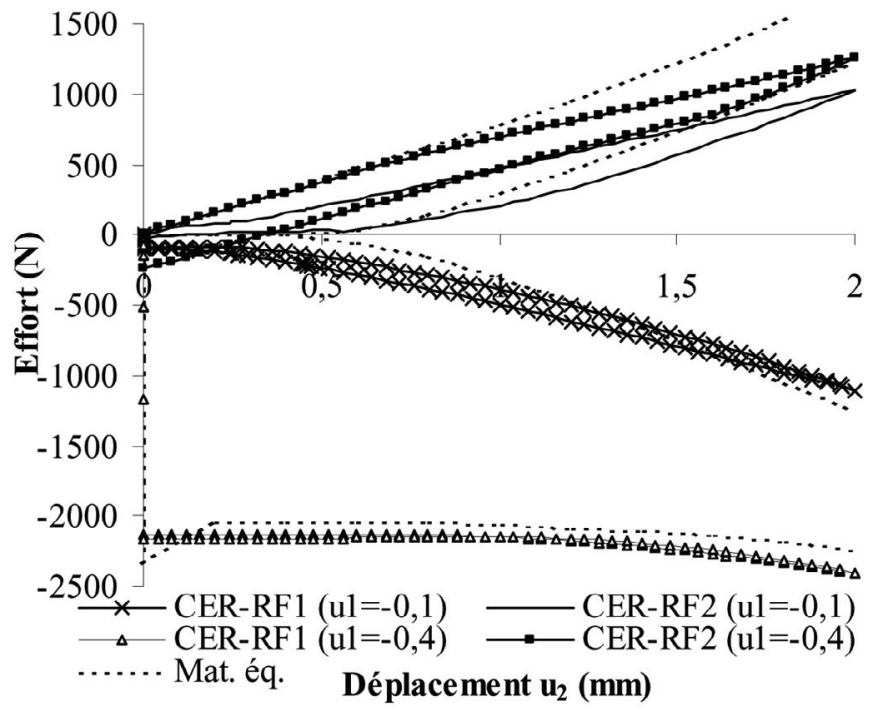

(b)

Fig. 12. Chargement de cisaillement dans la direction 2 (sous compression transverse) : (a) conditions aux limites pour la simulation sur la cellule élémentaire; (b) comparaison des courbes obtenues avec la cellule élémentaire (CER) et avec le matériau équivalent (Mat. Éq.) pour deux valeurs de compression transverse différentes $u_{1}$ de 0,1 et $0,4 \mathrm{~mm}$ (RF1 et $\mathrm{RF} 2$ sont les forces de réaction dans les directions 1 et 2 , respectivement).

dans les deux directions (voir Fig. 13). Les valeurs obtenues sont :

$$
\begin{aligned}
\sigma_{1}^{0} & =-5,5 \times 10^{-4} \mathrm{MPa} \\
\sigma_{1}^{\mathrm{f}} & =-5 \mathrm{MPa} \\
\sigma_{2} & =-1,1 \times 10^{-3} \mathrm{MPa}
\end{aligned}
$$

Les simulations de chargements de compression, traction et cisaillement avec le modèle simplifié permettent d'évaluer son domaine de validité. Les simulations de compression dans les deux directions principales (Fig. 9) montrent une bonne cohérence avec les courbes obtenues sur la cellule élémentaire. La seule différence se situe dans la première partie non-linéaire qui n'est pas reproduite 
Tableau 1.

\begin{tabular}{cll}
\hline & Joints ouverts (direction 1) & Joints fermés (direction 1) \\
\hline & $E_{1}=0 \mathrm{MPa}$ & $E_{1}=7000 \mathrm{MPa}$ \\
Joints ouverts & $E_{2}=0 \mathrm{MPa}$ & $E_{2}=0 \mathrm{MPa}$ \\
(direction 2) & $\nu_{12}=0$ & $\nu_{12}=0,2$ \\
& $G_{12}=0 \mathrm{MPa}$ & $G_{12}=1000 \mathrm{MPa}$ \\
\hline \multirow{3}{*}{ Joints fermés } & $E_{1}=3700 \mathrm{MPa}$ & $E_{1}=7000 \mathrm{MPa}$ \\
(direction 2) & $E_{2}=7000 \mathrm{MPa}$ & $E_{2}=7000 \mathrm{MPa}$ \\
& $\nu_{12}=0,11$ & $\nu_{12}=0,2$ \\
& $G_{12}=1000 \mathrm{MPa}$ & $G_{12}=2900 \mathrm{MPa}$ \\
\hline
\end{tabular}

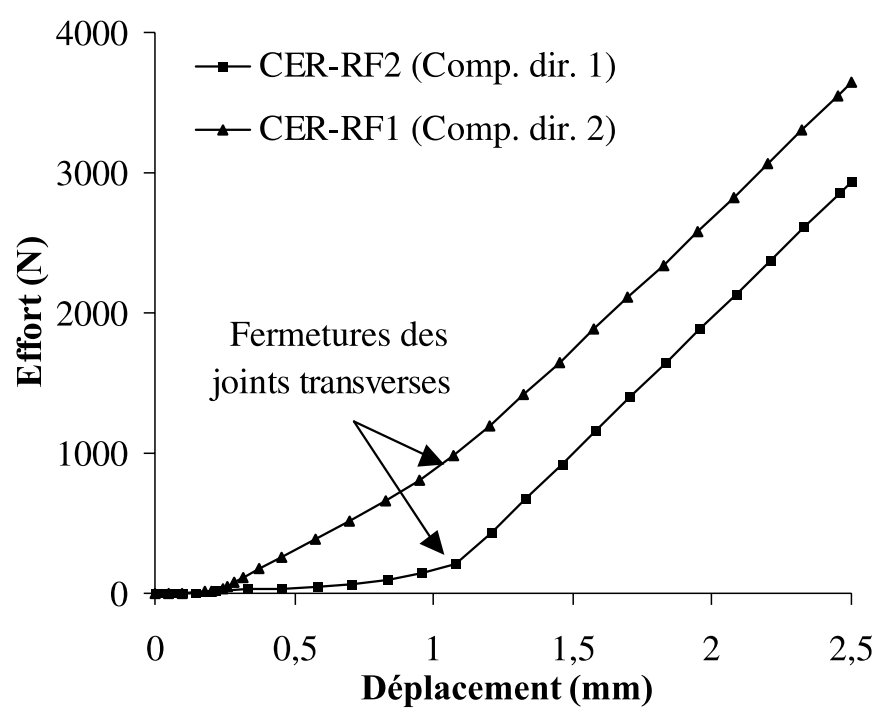

Fig. 13. Comportements transverses au cours de chargements de compression dans les directions 1 et 2 (cellule élémentaire), avec détermination de la fermeture des joints transverses (RF1 et $\mathrm{RF} 2$ sont les forces de réaction dans les directions 1 et 2 , respectivement).

par le matériau équivalent, car les joints sont soit ouverts, soit fermés, sans cas intermédiaire.

En ce qui concerne les simulations du chargement de traction (Fig. 10), le modèle ne permet d'obtenir que la première partie linéaire qui correspond à une étape sans glissement. Ceci représente une limitation du modèle.

Enfin, les simulations des chargements de cisaillement (Figs. 11 et 12) montrent que le modèle donne des résultats qui ne sont pas trop éloignés de ceux sur la cellule élémentaire, et ceci en dépit de la relative dispersion des courbes obtenues sur cette cellule élémentaire.

Du fait que ce modèle sera utilisé en température, un chargement thermique a alors été simulé sur la cellule élémentaire, et avec le matériau équivalent. Il s'agit d'un chargement uniforme en température, qui évolue de $20^{\circ} \mathrm{C}$ à $400{ }^{\circ} \mathrm{C}$, appliqué sur la cellule élémentaire encastrée sur ses quatre bords. La comparaison de l'évolution des efforts sur les bords, dans les deux directions, montre une bonne cohérence des résultats entre le modèle simplifié et la cellule élémentaire (Fig. 14).

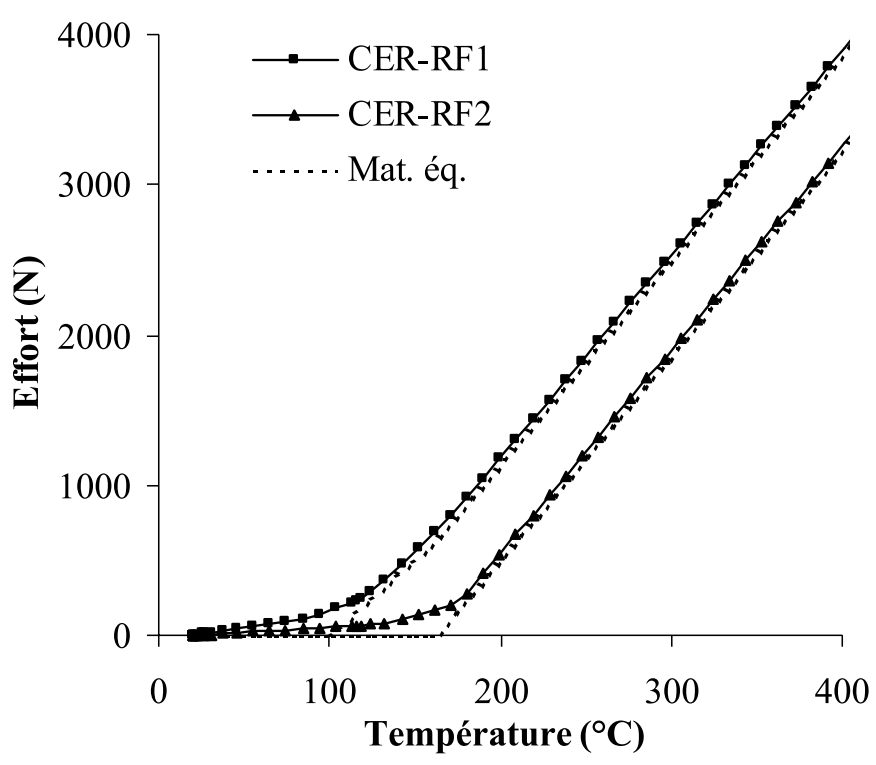

Fig. 14. Chargement thermique (température uniforme appliquée sur toute la cellule encastrée sur ses quatre bords) : comparaison des courbes obtenues sur la cellule élémentaire (CER) et avec le matériau équivalent (Mat. Éq.) (RF1 et RF2 sont les forces de réaction dans les directions 1 et 2 , respectivement)

\section{Validation}

\subsection{Essai thermomécanique}

Un essai thermomécanique a été réalisé par la société Sollac [22]. Il concerne une structure métallique cylindrique (diamètre : $2 \mathrm{~m}$, épaisseur : $30 \mathrm{~mm}$ ) ayant un fond plat recouvert d'une maçonnerie (constituée de briques en bauxite cuites) présentée dans la figure 15. La structure métallique et la maçonnerie sont séparées par un joint de béton réfractaire de $30 \mathrm{~mm}$ d'épaisseur. La partie supérieure de la maçonnerie plane est soumise à un chargement thermique uniforme appliqué progressivement $\left(50{ }^{\circ} \mathrm{C} / \mathrm{h}\right)$ jusqu'à $1500^{\circ} \mathrm{C}$. La structure métallique est refroidie à $20^{\circ} \mathrm{C}$ sur sa partie extérieure. Des jauges de déformation, collées sur la face externe de la structure métallique aux points $\mathrm{A}, \mathrm{A}^{\prime}, \mathrm{B}$ et $\mathrm{B}^{\prime}$, permettent de déterminer, par exemple, les contraintes tangentielles 
A

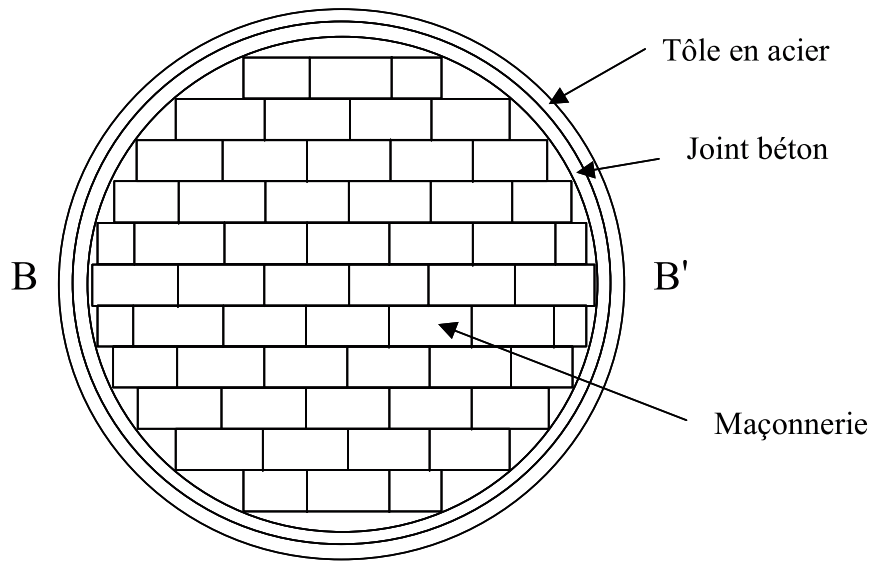

$\mathrm{A}^{\prime}$

Fig. 15. Schéma de la maçonnerie plane.

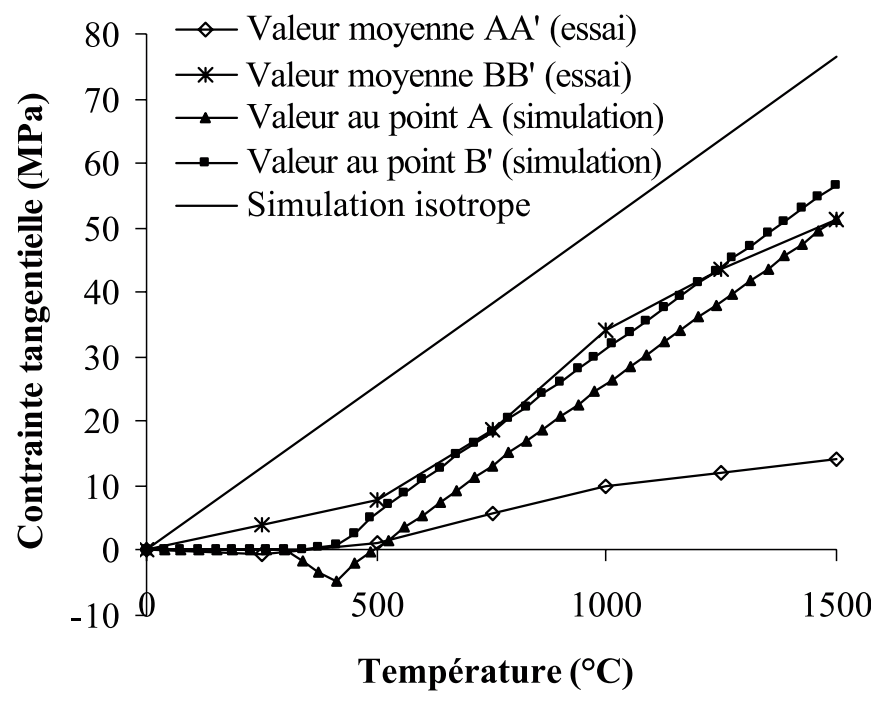

Fig. 16. Contraintes tangentielles sur la face extérieure de la structure métallique : valeurs moyennes expérimentales (aux points $\mathrm{AA}^{\prime}$ et $\mathrm{BB}^{\prime}$ ), valeurs numériques aux points $\mathrm{A}$ et $\mathrm{B}^{\prime}$ (simulation avec le matériau équivalent), et simulation isotrope (sans joints).

(Fig. 16). Une anisotropie des résultats (due à la présence d'un nombre de joints différent dans les deux directions) est observée.

\subsection{Simulation}

L'essai présenté ci-dessus a été simulé en 2D (en contraintes planes) en remplaçant la maçonnerie par le matériau équivalent simplifié. Seul un quart de la structure a été modélisé (à cause des symétries, Fig. 17). Dans un but de simplification, l'acier, le béton réfractaire et la maçonnerie sont supposés parfaitement liés.

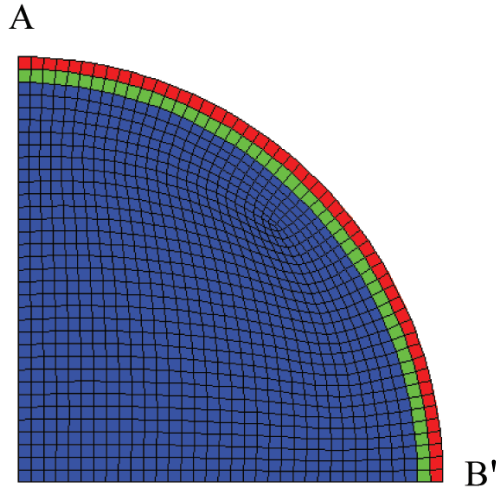

Fig. 17. Maillage du quart de la structure.

Deux problèmes apparaissent dans cette modélisation très simplifiée :

- quel champ de température faut-il appliquer en 2D alors que le champ réel est 3D (la température n'étant pas constante dans l'épaisseur de la brique)?

- comment prendre en compte le fait que certains vides existent entre la maçonnerie et le joint en béton réfractaire (voir Fig. 15) à cause de la forme rectangulaire des briques, alors que le matériau équivalent est complètement en contact avec ce joint?

Le champ de température a été choisi comme étant celui imposé sur la partie supérieure des briques (c'est celui qui semble être le meilleur champ 2D) : une température de $1500{ }^{\circ} \mathrm{C}$ est imposée sur la maçonnerie et le joint en béton, et $20{ }^{\circ} \mathrm{C}$ sur la face extérieure de la structure métallique (ainsi la température décroît linéairement dans l'épaisseur de l'acier).

Les vides présents entre les briques et le joint en béton sont pris en compte en donnant un comportement très mou au matériau du joint. Son module d'Young (30 MPa) a été déterminé de telle manière qu'une simulation isotrope (avec un comportement isotrope linéaire pour le matériau équivalent) donne la rigidité de l'essai (voir Fig. 16).

La simulation réalisée avec le matériau équivalent (Fig. 16) permet de retrouver certains résultats expérimentaux :

- un niveau de contrainte plus faible que pour la simulation isotrope (réalisée avec le comportement de la brique, sans joints) montrant l'influence des joints,

- un comportement anisotrope entre les deux directions du fait qu'il y a deux fois plus de joints dans la direction $\mathrm{AA}^{\prime}$ que dans la direction $\mathrm{BB}^{\prime}$.

La différence observée entre les courbes expérimentales et numériques au point $\mathrm{A}$ peut être expliquée par la simplicité du modèle numérique (en 2D), et également par le comportement élastique linéaire pris pour le matériau des briques (qui, en réalité, devient viscoélastique à haute température). 


\section{Conclusions}

Afin de prendre en compte l'effet des joints dans les maçonneries réfractaires, ces maçonneries sont remplacées, dans un calcul par éléments finis, par un matériau équivalent. Un premier modèle simplifié a été présenté en 2D. Le comportement du matériau est élastique orthotrope non-linéaire, dépendant de l'état d'ouverture ou de fermeture des joints. Ses paramètres ont été identifiés à l'aide d'une méthode inverse, à partir de différents chargements simulés avec un modèle défini à l'échelle des composants (briques et joints). Cette première approche multiéchelles a été validée par un essai thermomécanique sur une structure contenant une maçonnerie plane.

L'avantage de ce modèle simplifié est qu'il peut être facilement utilisable dans des conditions industrielles, tout en donnant des résultats relativement satisfaisants dans le cas où les glissements entre briques sont faibles.

Pour la poursuite de l'étude, le modèle doit être amélioré et développé dans les directions suivantes :

- la modélisation 3D,

- la fermeture progressive des joints (évolution progressive du module d'Young en fonction des déformations),

- la prise en compte du glissement (qui peut être modélisé par un comportement plastique parfait),

- les maçonneries cylindriques (non planes),

- l'application à des structures réelles, telles des poches à acier (avec des maçonneries planes et cylindriques).

Remerciements. Les auteurs tiennent à remercier le groupe Arcelor (Sollac Dunkerque) pour son soutien.

\section{Références}

[1] E.D. Gordon, Refractories in CFB applications, Proceedings of 12th International Conference on Fluidized Bed Combustions, San Diego, USA, 1993, pp. 967-983

[2] J. Poirier, Recent tendencies in refractories in relation with service in the steel industry, Proceedings of 39th Colloquium on Refractories, Aachen, Germany, 1996, pp. $6-16$

[3] S. Peruzzi, J. Poirier, J.C. Glandus, M. Huger, Numerical study of the in-serve behaviour of refractory parts used in continuous casting, Proceedings of 6th European Ceramic Society Conference, Brighton, UK, 1999, pp. 161-162

[4] H.P. Lee, Thermal stress analyses of a waste storage container, Computers and Structures 61(2) (1996) 197-224

[5] R. Stuart, L. Shipley, A. Ghose, M. Hiremath, Thermal degradation of the concrete roof of high-level waste storage tank, Computers and Structures 64(5-6) (1997) 959-972
[6] J.F. Hernandez, N. Schmitt, P. Meunier, J. Poirier, Thermomechanical computations of refractory structure. Improvement due to the modelling of the dehydration process in high alumina castable, Proceedings of UNITECR'99, Berlin, Germany, 1999, pp. 107-109

[7] V. Derré, A. Gasser, P. Boisse, Poches à acier 270 tonnes à tenue améliorée, Rapport Usinor/LMSP, Orléans, France, 2000

[8] A. Anthoine, Derivation of the in-plane elastic characteristics of masonry through homogenization theory, Int. J. Solids Structures 32(2) (1995) 137-163

[9] P. Pegon, A. Anthoine, Numerical strategies for solving continuum damage problems with softening: application to the homogenization of masonry, Computers and Structures 64(1-4) (1997) 623-642

[10] A. Cecchi, R. Di Marco, Homogenization of masonry walls with a computational oriented procedure. Rigid or elastic block? Euro. J. Mechanics - A/Solids 19(3) (2000) 535-546

[11] G. Ma, H. Hao, Y. Lu, Homogenization of masonry using numerical simulations, J. Eng. Mechanics 127(5) (2001) 421-431

[12] A. Zucchini, P.B. Lourenco, A micro-mechanical model for the homogenisation of masonry, Int. J. Solids Structures 39(12) (2002) 3233-3255

[13] K. Takahasi, Y. Miyamoto, M. Kumagai, Thermomechanical stress analysis in brick linings by FEM using non-linear boundary condition, Proceedings of UNITECR'97, New Orleans, USA, 1997, pp. 349-357

[14] K. Andreev, H. Harmuth, Modelling of the thermomechanical behaviour of the lining material of teeming ladles, Proceedings of UNITECR'01, Cancun, Mexico, 2001, CDRom

[15] K. Levenberg, A method for the solution of certain nonlinear problems in least squares, Quart. Appl. Math. 2 (1944) 164-168

[16] D.W. Marquardt, An algorithm for least squares estimation of nonlinear parameters, J. Soc. Indus. Appl. Math. 11(2) (1963) 431-441

[17] D.S. Schnur, N. Zabaras, An inverse method for determining elastic material properties and a material interface, Int. J. Numerical Methods Eng. 33 (1992) 2039-2057

[18] A. Gasser, P. Boisse, Y. Dutheillet, J. Poirier, Experimental and numerical analyses of thermomechanical refractory lining behaviour, J. Materials: Design and Application (IMechE), vol. 245, Part L, 2001, pp. 41-54

[19] M. Sutton, W.J. Wolters, W.H. Peters, W.F. Ranson, S.R. McNeill, Determination of displacements using an improved digital correlation method, Image \& Vision Computing 1-3 (1983) 133-139

[20] P. Vacher, S. Dumoulin, R. Arrieux, Determination of the forming limit diagram from local measurement using digital image analysis, Int. J. Forming Processes 2(3-4) (1999) 395-408

[21] F. Dumont, G. Hivet, R. Rotinat, J. Launay, P. Boisse, P. Vacher, Mesures de champ pour des essais de cisaillement sur des renforts tissés, Mécanique \& Industries 4 (2003) 627-635

[22] D. Themines, Comportement des maçonneries, Rapport Sollac, Dunkerque, France, 1994 